MATEC Web of Conferences 48,05001 (2016)

DOI: $10.1051 /$ matecconf $/ 20164805001$

C) Owned by the authors, published by EDP Sciences, 2016

\title{
Computational approach to assess postural tests under microgravity conditions
}

\author{
Konstantin Brazovskii ${ }^{1, a}$, Vasilii Fokin ${ }^{2}$, Ivan Tolmachev ${ }^{2}$, Jacov Pekker ${ }^{2}$ and David Hachaturyan ${ }^{1}$ \\ ${ }^{1}$ National Research Tomsk Polytechnic University, 634050 Tomsk, Russia \\ ${ }^{2}$ Siberian State Medical University, 634050 Tomsk, Russia
}

\begin{abstract}
Human vestibular system is extremely fragile and rapidly degrades when gravity is significantly lower than the gravity of Earth. Unfortunately, current balance assessment tools, including state-of-start computerized centre-of-mass measurement platforms, cannot be used in space because of the low gravity. Thus, a problem of developing new methods to assess postural stability under microgravity conditions is very important. This paper describes a distance-based criterion to estimate multidimensional measurements and its application for postural tests assessment. It has been shown that proposed criterion may be effectively used to estimate the equilibrium function by means of low-end hardware, which does not rely on the gravitational force. The criterion was compared against several known indexes and its ability to distinguish disorders of the balance has been studied.
\end{abstract}

\section{Introduction}

Human vestibular system demonstrates substantially disturbed behaviour under microgravity conditions and should be monitored constantly during long term space travels. The influence of microgravity on human's sense of balance has been studying for many years, and eventually it became clear that maintaining the equilibrium function in appropriate functional state is extremely important to prevent development of serious disorientation and neurological disorders.

An ability to balance and maintain postural stability is an essential diagnostic criterion of a wide range of disorders. A great variety of tests, functional and quantitative as well, has been developed since the Romberg manoeuvre was introduced in the middle of the XIX century. The general idea of conventional postural tests data processing methods is to estimate statistical parameters of movements under certain conditions, and then summarize the raw values into scores. This approach has been clinically validated and, that is also important, is based on a quite simple numerical procedure with clear interpretation.

The current "gold standard" quantitative test is an analysis of the centre of pressure (COP) by means of high precision computerized force plates [1]. The COP is quite sensitive to Parkinson's disease (PD) [2], even in an early stage of PD, and also to other balance disorders. Unfortunately, it has been reported [3] that the COP method has some disadvantages, such as a large size of equipment, which should be properly installed in stationary conditions and thoroughly calibrated before use. One more issue of the posturography is a requirement that all force platforms intended for clinical

${ }^{\mathrm{a}}$ Corresponding author : bks_2005@mail.ru

This is an Open Access article distributed under the terms of the Creative Commons Attribution License 4.0, which permits unrestricted use, distribution, and reproduction in any medium, provided the original work is properly cited. 
applications should satisfy the standards of the International Society for Posture and Gait Research (ISPGR), it means that the devices must be able to measure movements and displacements with $\pm 0,1$ $\mathrm{mm}$ accuracy and $0,05 \mathrm{~mm}$ resolution. Such a high level of metrological characteristics is achievable, but for a high price. As a result, the computerized posturography (CPG) is very useful but relatively expensive method, which can be used effectively to resolve some specific clinical issues rather than on a daily basis. Unfortunately, widely used conventional hardware to evaluate a functional state of the equilibrium sense is strongly depended on the precise value of the gravitational force, thus cannot be used under microgravity conditions.

Reportedly, traditional neurological tests do not work in space because they are tightly linked to gravity [4]. The experimental setup without dependency on the precise value of gravity, proposed in [4], demonstrates impressive diagnostics capability for the price of more complex design and sophisticated data processing techniques.

Taking into account possible consequences of postural instability and balance disorders during long term space travels, the development of valid balance assessment tools usable in space, is undoubtedly important [4]. Recently quite a few hardware tools and computational methods to collect and process data of postural tests have been introduced; primarily for aerospace applications, which means high price and limited availability. The objective of the presented study, inspired by the current aerospace technologies, is to develop a quantitative method to assess maintaining postural stability during functional tests, which could be used under low gravity conditions.

\section{Study design}

The study was conducted in two groups: the first, reference group, consisted of 9 healthy subjects; the second group was comprised of 9 patients with minor neurological deficits and negative Romberg's test.

Functional postural tests were used along with a video-based markless motion capture technique. The protocol included Romberg's test that followed by the Balance Evaluation Systems Test (miniBESTtest, [4]). The Cartesian coordinates of the featured points were captured by means of Microsoft Kinect hardware. The "Brekel Kinect free" software was used to preprocess and collect raw data.

Finally, our new numerical distance-based criterion was calculated. After that the criterion was compared against the scores of the BESTtest and known indexes, such as average speed and patented Equilibrium Function Quality (EFQ) [6]. Statistical hypotheses were tested by Mann-Whitney U nonparametric test (MW test), Spearmen rank correlation was used as well.

\subsection{Materials and methods}

The theoretical details of the criterion were published in [7]. Briefly, the data processing pipeline can be given as follow:

1. First of all, time series vectors $\mathbf{x}(t), t=0, \ldots, T$ have to be measured for each subject during the period of time with length $T$. Here $\mathbf{x}(t)$ is a multidimensional value, for example, the $3 \mathrm{D}$ coordinates of a certain point.

2. The whole interval $[0, T]$ then is divided into $k$ fragments with length $\Delta T$. The average values $\mathbf{x}_{\mathrm{j}}$, $j=0, \ldots, k$ are calculated inside the $[t, t+\Delta T]$ intervals (1):

$$
\mathbf{x}_{j}=\frac{1}{n} \sum_{i=1}^{n} \mathbf{x}\left(t_{i}\right)
$$

where $n$ is a number of the measured values inside the interval.

3. The averaged values are normalized (2) and put into the $N$ x $k$ state matrix $S, N$ is a number of subjects in a certain group. 


$$
\begin{aligned}
& \mathbf{x}_{j, \text { norm }}=\frac{\mathbf{x}_{j}-\mathbf{x}_{\text {min }}}{\mathbf{x}_{\text {max }}-\mathbf{x}_{\text {min }}}, j=1, k \\
& \mathbf{x}_{\text {min }}=\min _{j=1, k}\left\{\mathbf{x}_{j}\right\}, \quad \mathbf{x}_{\text {max }}=\max _{j=1, k}\left\{\mathbf{x}_{j}\right\}
\end{aligned}
$$

4. All averaged and normalized values of the healthy subjects $\mathbf{x}_{\mathrm{V}}$ are merged into the reference matrix $\mathbf{S}_{0}$ with size $N_{0}$, and then a covariance matrix $\mathbf{C}$ with $k \times \mathrm{x} k$ size is calculated.

5. Finally, the distance-based dimensionless criterion $I d$ is calculated (3), using the averaged and normalized values $\mathbf{x}_{\mathrm{u}}$ obtained from the patients.

$$
I d\left(\mathbf{x}_{u}\right)=\frac{1}{2 k}\left[\frac{1}{N_{0}} \sum_{v=1}^{N_{0}}\left(\mathbf{x}_{u}-\mathbf{x}_{v}\right) \mathbf{C}^{-1}\left(\mathbf{x}_{u}-\mathbf{x}_{v}\right)\right]
$$

where $\mathbf{x}_{\mathrm{v}}$ are the reference vectors from the reference group, and $\mathbf{x}_{\mathrm{u}}$ are the test vector obtained from a certain patient, $\mathbf{C}^{-1}$ is an inverse covariance matrix. In other words, our criterion is the averaged squared Mahalanobis distance between the certain vector and all vectors from the reference set.

Also we used EFQ test, which is based on a vector statokinesigram and includes calculation of COP's velocity at each moment of time. After that a cumulative distribution function of the velocity distribution was approximated. The actual value of EFQ is relative area under the cumulative curve, which is given as a percentage.

Usually, the conventional methods are able to evaluate movement of a single point (centre of pressure, for example) only. In contrast, the $I d$ is not limited by the dimension of the measured vectors and almost any reasonable length is appropriate. In order to make the developed criterion comparable to the common ones, a point corresponding to the center of mass was chosen. Vertical displacement $(z$ coordinate) was omitted. The data were recorded during Romberg's test with $15 \mathrm{samples} / \mathrm{second}$ rate and estimated spatial resolution about one millimetre. The overall period of time was $T=10 \mathrm{sec}$, $\Delta T=2 \mathrm{sec}, n=30$.

Table 1. Individual parameters for the studied subjects.

\begin{tabular}{|c|c|c|c|c|}
\hline Subject & $\begin{array}{c}\text { MiniBEST test } \\
\text { scores }\end{array}$ & $\begin{array}{c}\text { Average speed } \\
\text { Me }[\mathrm{Q} 0,25-\mathrm{Q} 0,75] \mathrm{m} / \mathrm{sec}\end{array}$ & EFQ, \% & Id \\
\hline R1 & 27 & $0,103[0,056-0,159]$ & 81,34 & 1,63 \\
\hline R2 & 28 & $0,015[0,008-0,021]$ & 73,83 & 1,37 \\
\hline R3 & 28 & $0,057[0,033-0,096]$ & 85,23 & 1,21 \\
\hline R4 & 28 & $0,013[0,008-0,021]$ & 81,01 & 1,15 \\
\hline R5 & 27 & $0,034[0,019-0,063]$ & 83,89 & 1,30 \\
\hline R6 & 28 & $0,021[0,010-0,032]$ & 76,04 & 1,35 \\
\hline R7 & 27 & $0,040[0,021-0,082]$ & 83,36 & 1,37 \\
\hline R8 & 27 & $0,057[0,028-0,100]$ & 84,36 & 1,46 \\
\hline R9 & 27 & $0,058[0,030-0,103]$ & 82,95 & 1,66 \\
\hline P1 & 13 & $0,083[0,039-0,164]$ & 93,62 & 6,97 \\
\hline P2 & 11 & $0,015[0,010-0,022]$ & 91,21 & 7,52 \\
\hline P3 & 28 & $0,015[0,010-0,022]$ & 91,01 & 3,07 \\
\hline P4 & 28 & $0,024[0,014-0,058]$ & 86,58 & 9,72 \\
\hline P5 & 15 & $0,040[0,020-0,060]$ & 76,51 & 2,33 \\
\hline P6 & 28 & $0,022[0,012-0,041]$ & 93,86 & 5,23 \\
\hline P7 & 26 & $0,030[0,021-0,046]$ & 67,65 & 1,82 \\
\hline P8 & 28 & $0,012[0,008-0,020]$ & 78,19 & 1,65 \\
\hline P9 & 28 & $0,020[0,011-0,032]$ & 82,55 & 3,46 \\
\hline MW test & $p=0,43$ & $p=0,54$ & $p=0,38$ & $p<0,001$ \\
\hline Ref vs Pat & & & & \\
\hline
\end{tabular}




\section{Results}

The individual calculated parameters are given in Table 1. The subjects R1-R9 form the reference group (Ref), and P1-P9 are in the group of patients (Pat). The average speed is given as a median and quartiles because this parameter does not fit the normal distribution.

Despite the fact that all patients were Romberg's negative, three of them had the miniBESTtest scores from 11 to 15 . Nevertheless, the difference between two groups (reference and patients) is not statistically significant.

The average speed and EFQ also have practically the same values in both groups, whereas the distance-based criterion $I d$ substantially varies between groups. What is more important is that all these parameters are not correlated (Spearmen $R$ absolute values are less than 0,33 with $p>0,15$ ).

The example of the raw data ( $X$ and $Y$ coordinates of the selected point against time and its scattergram) is plotted in Figure 1. The displacements are within the $10 \mathrm{~mm}$ range, and scattergrams have no signs of imbalance. The cumulative percentages related to the EFQ index are shown in Figure 2. The cumulative distribution function of speed of the point was approximated by means of the empirical distribution step function.

The simplest way to explain the featured properties of our distance-based criterion $I d$ is to represent the subjects, which involved in the study, as a set of point in the 3D space of per-coordinate components $I d x, I d y, I d z$ (Figure 3). Note, that the scales of axis are different and the $I d x, I d y, I d z$ are the square root components of $I d$ in (3). It is clear that the reference group is quite compact, it members form one distinguishable cluster, while the patients are spread at a quite long distance from the reference group. This finding, on the one hand, highlights an inhomogeneity of the patient's group. On the other hand, the location of the certain patient may be considered either as an individual characteristic or as a pathological sign.
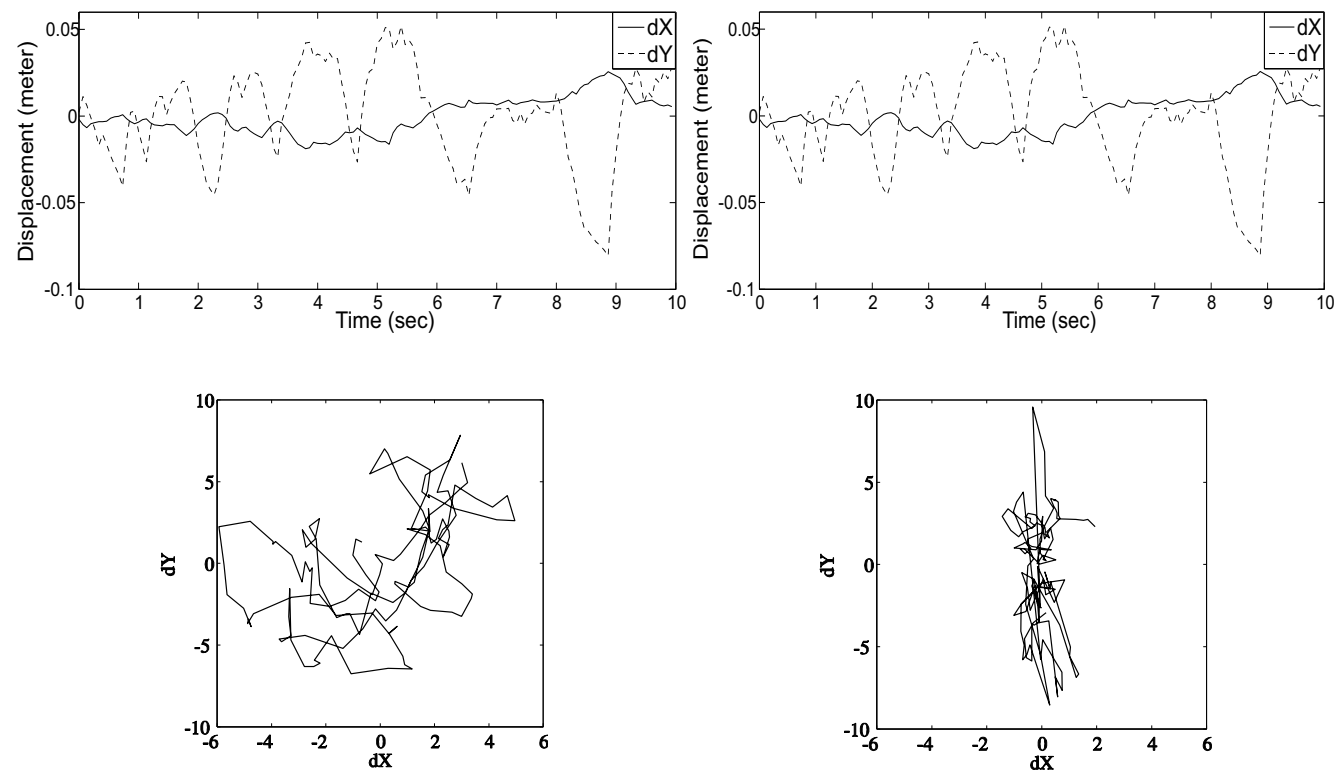

Figure 1. The displacements (upper plots) and scatterogram (lower plots) of the selected point for the healthy subject R2 (left plot) and patient P1 (right plot). 

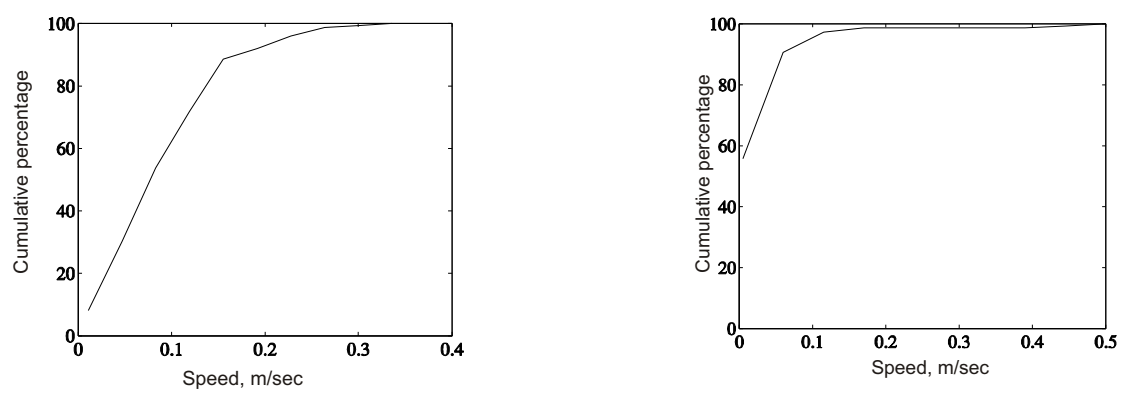

Figure 2. The cumulative percent of speed of the selected point for the healthy subject R2 (left plot) and patient P1 (right plot).

\section{Discussion}

The main result of our study is a simple and quite sensitive distance-based criterion to assess dissimilarity between, at least, two sets of measured values. The criterion mainly relays on the wellknown Mahalanobis metric and inherited the ability to deal with correlated noisy data. This feature of the criterion is very important, because the key measurable parameters of the motion (positions and speed of the selected points) seem to be mutually linked. This may be a reason why some conventional methods, which imply statistical independence of the parameters, sometimes fail to estimate state of imbalance [8]. Another problem with these methods is that they are highly sensitive to noisy and inaccurate data. The distance-based approach has an innate ability to average chaotic signals and decrease noise proportionally to the square root of a vector's length [9]. We exploit this feature in a slightly different manner: the criterion requires a large reference group in order to achieve a desired confidence level, according to the formula (3). This feature may be considered as a drawback, but in practice of clinical studies a control (or reference) group of an appropriate size is an essential part of the research design. However, members of the reference group should be carefully selected.

The positive side-effect of the distance-based approach is that it reduces technical requirements to measuring devices. The accuracy and resolution of the motion capture hardware, which was used in our study, are sufficiently lower in comparison with the state-of-art force plates, but even in that case it was possible to distinguish between the groups.

\section{Conclusion}

A new approach to acquire and process data of postural tests has been proposed and proved to be sensitive under normal gravity conditions. The method takes into account small variations of the trajectory linked to individual physiological features of the vestibular system. A data gathering procedure does not involve the exact value of gravity, thus could be possible used under microgravity conditions. The main advantages of the approach are outstanding simplicity and low cost of hardware along with a high level of sensitivity. The specificity of the developed criterion towards the certain vestibular pathology is a discussable point and should be further investigated.

\section{References}

1. M. E. Pedalini, O. L. Cruz, R.S. Bittar, M.C. Lorenzi, S.S. Grasel, Acta Otolaryngol 129(9), 962 (2009)

2. N. Chastan, B. Debono, D. Maltete, J. Weber, Mov Disorders 23, 366 (2008)

3. M. Mancini, F.B. Horak, E J Phys Med Rehabil 46(2), 239 (2010) 
4. C. Casellato, M. Tagliabue, A. Pedrocchi, C. Papaxanthis, G. Ferrigno, T. Pozzo, Exp Brain Res 216, 203 (2012)

5. M. Visser, J. Marinus, B.R. Bloem, H. Kisjes, B.M. van den Berg, J.J. van Hilten, Arch Phys Med Rehabil 84(11), 1669 (2003)

6. Computer stabiloanalyzer with biofeedback. URL: http://www.scenar.com.ru/production /stabile/index.htm (2016)

7. V.A. Fokin, Bull. TPU 311(5), 120 (2007)

8. R.J. Schilling, E.M. Bollt, G.D. Fulk, J.D. Skufca, A.F. Al-Ajlouni, C.J. Robinson, IEEE Trans. Biomed. Eng. 56(2), 292 (2009)

9. P. Nicolas, B-H. Avner, J. Classif 29(1), 23 (2012) 